\title{
Evaluation of a New Oral Appliance With Objective Compliance Recording Capability: A Feasibility Study
}

\author{
Jerry Hu, DDS ${ }^{1}$; Len Liptak, MBA² \\ ${ }^{1}$ Jerry Hu Family Dentistry, Soldotna, Alaska; ${ }^{2}$ ProSomnus Sleep Technologies, Pleasanton, California
}

\begin{abstract}
Study ОвJестіves: The aim of this study is to evaluate the clinical feasibility of a novel oral appliance therapy device with a compliance recorder (OAT-CR), and report the objectively collected data. This is the first study to report on a commercially available OAT-CR.

MethoDs: This was a single-center pilot study that utilized an intercept case format. Eight consecutive patients from the practice, in whom obstructive sleep apnea was already diagnosed and who were treated with standard of care oral appliance therapy, were enrolled in the study. Each participant was fitted with a new OAT-CR. The objectively recorded compliance data were acquired at routine follow-up appointments.
\end{abstract}

RESULTS: The OAT-CR device worked as intended for eight of eight patients. The objectively recorded compliance rate in this study was $87.9 \% \pm 20.4 \%$. Mean usage was $7.4 \pm 1.4$ hours per night, based on the objectively recorded compliance data.

Conclusions: Based on the results for this pilot study, the new OAT-CR is an option for clinical situations where objective compliance tracking is required or preferred. Objectively recorded compliance and mean nightly usage data in this study affirm oral appliance therapy compliance rates reported in previous studies.

KEYWORDS: compliance, objective compliance recorder, obstructive sleep apnea, oral appliance, oral appliance therapy, sleepdisordered breathing

Citation: Hu J, Liptak L. Evaluation of a new oral appliance with objective compliance recording capability: a feasibility study. Journal of Dental Sleep Medicine. 2018;5(2):47-50.

\section{INTRODUCTION}

Obstructive sleep apnea (OSA) is a medical condition associated with significant health risks. ${ }^{1}$ Compliance is considered an important determinant of therapeutic effectiveness. ${ }^{2}$ Improvements in symptoms, daytime sleepiness, quality of life, and other patient health and wellness attributes have been observed when patients comply with therapy. ${ }^{3}$ Conversely, intermittent compliance, or compliance below recommended levels, has been shown to obviate improvements. ${ }^{4-6}$

There are indications that differences in compliance rates explain the similarities in health outcomes between oral appliance therapy (OAT) and continuous positive airway pressure (CPAP), despite the superior efficacy of CPAP. ${ }^{7,8}$ Mean hours of use per night are reported to be higher with OAT than CPAP. Compliance rates are reported to be higher for OAT than CPAP.

The quality of evidence supporting the superior compliance associated with OAT is, however, considered to be low. ${ }^{9}$ Most studies reporting OAT compliance rates have been predominantly based on subjective reporting methods such as treatment diaries. Three papers have been published that feature OAT devices with compliance recording (OAT-CR) technologies. ${ }^{10-12}$ However, there remains a call for more objectively recorded data, and none of the previously published papers evaluate OAT-CR technologies that are readily available to everyday dental sleep medicine practitioners. For example, this is the first paper to report on an OAT-CR that has been cleared for market by the United States Food and Drug Administration (FDA). ${ }^{13}$
Thus, the primary aim of this study is to evaluate the feasibility and survivability of a commercially available OAT-CR (ProSomnus IA Sleep Device, ProSomnus Sleep Technologies, Pleasanton, California, United States with the Dentitrac Compliance Recorder, Braebon Medical Corporation, Kanata, Ontario, Canada). The secondary aim for this study is to report the objectively recorded compliance data from this study population.

\section{METHODS}

This single-center pilot study utilized an intercept case protocol. The intercept protocol consisted of enrolling patients already in OAT and fitting them with the new OAT-CR. The intercept method was selected to avoid subjecting the patient to additional procedures or any gaps in therapy; the new OAT-CR was fabricated from the digital impression and digital bite records on file at the manufacturer. The intercept method was also selected to enable patients to provide more insightful feedback on device preference, acceptance, and overall experience.

Eight consecutive patients, already scheduled for follow-up appointments, were invited to participate in the study. Each of these patients received a previous diagnosis of OSA by a board certified medical doctor, and were already undergoing standard of care OAT. Participants were informed and provided consent. However, participants were not aware that compliance recording was the purpose of the change in oral appliance.

Four men and four women were enrolled into the study. The average age of the participants was $54.6 \pm 12.8$ years. The 
Table 1-Study population characteristics $(n=8)$.

\begin{tabular}{|l|c|}
\hline Sample Characteristics & \\
\hline Age (years) & $54.6 \pm 12.8$ \\
\hline Height $(\mathrm{cm})$ & $173.6 \pm 7.3$ \\
\hline Weight $(\mathrm{lb})$ & $178.8 \pm 29.0$ \\
\hline BMI $\left(\mathrm{kg} / \mathrm{m}^{2}\right)$ & $25.7 \pm 5.3$ \\
\hline Sex $($ male / female) & $4 / 4$ \\
\hline
\end{tabular}

Values are mean \pm standard deviation. $\mathrm{BMI}=$ body mass index.

average body mass index was $25.7 \pm 5.3 \mathrm{~kg} / \mathrm{m}^{2}$. See Table 1 for characteristics of the study population. Each patient received a previous diagnosis by a medical professional and were undergoing standard of care OAT.

Pretreatment mean apnea-hypopnea index (AHI) for the overall study population was $37.2 \pm 41.4$ events/h. Two of the participants presented with mild OSA (AHI $<15$ events/h), three presented with moderate OSA (AHI 15 to 30 events/h), and three presented with severe OSA (AHI $>30$ events/h). With OAT, the mean AHI for the study population was $12.8 \pm 13.7$ events/h, representing an overall AHI improvement of $65.7 \%$ (Figure 1).

Seating of each OAT device and initialization of the compliance recorder was performed according to the provided instructions for use. The clinician confirmed fit, function, and comfort of the new oral appliance before releasing each patient. Compliance data were acquired from the devices at each follow-up appointment. Patient preference surveys were administered at each initial and follow-up appointments.

\section{RESULTS}

The OAT-CR functioned as intended for eight of eight study participants. Participants accepted the device, and used it throughout the duration of the study. The dental team was able to deliver the device according to the provided instructions for use. During follow-up appointments, the OAT compliance data were recorded, transferred, and accessed in a report for each participant. Compliance data were recorded for a total of 366 nights, a mean of $45.8 \pm 26.3$ nights per study participant.

The average objectively recorded compliance rate in this study was $87.9 \% \pm 20.4 \%$, using the $4 \mathrm{~h} /$ night, 5 nights/wk, definition for compliance. ${ }^{6}$ The objectively recorded mean nightly use was $7.4 \pm 1.4 \mathrm{~h} /$ night.

Three participants noted that the OAT-CR was less comfortable (eg, "more bulky") than their previous device (sleep device without a compliance recording chip, ProSomnus Sleep Technologies, Pleasanton, California, United States). This feedback did not inhibit the participant's ability to complete the study protocol.

\section{DISCUSSION}

The lack of objective compliance recording has been identified as a barrier for broader acceptance of OAT. ${ }^{14}$ Unlike CPAP
Figure 1-Oral appliance therapy efficacy for study population.

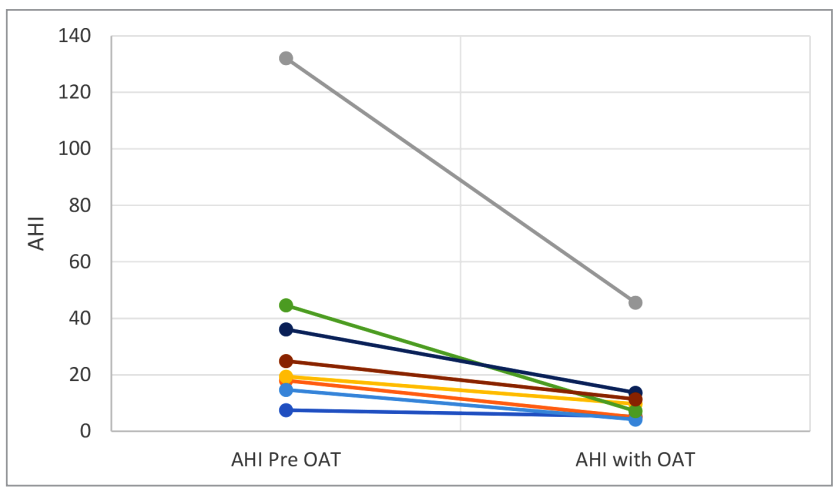

OAT = oral appliance therapy.

devices where compliance recording sensors are standard, there are few OAT devices with compliance recording capabilities. According to the FDA 510(k) Premarket Notification Database, 132 OAT devices have been cleared for market at the time this paper was authored. However, only four OAT-CRs have been cleared by the FDA. ${ }^{13}$ Thus, this is the first paper to evaluate performance of, and report data on, any of the compliance recorder enabled devices that have been cleared by the FDA.

The intercept case study method was successful in eliminating the need to subject participants to additional procedures and appointments. New devices with compliance chips were made from digital records. Each of the new devices fit, and were accepted by patients without major modifications. This is in agreement with prior studies that focused exclusively on the feasibility of a digitally fabricated oral appliance workflow. ${ }^{15}$ The OAT-CRs were manufactured in advance of the intercept appointments, enabling the patient to switch into the new device without a lapse in therapy.

The intercept method enabled study participants to compare experiences, given that the patients were successful in OAT without compliance recorders prior to the study. This was an important consideration. The compliance recorder chips added to the size and overall shape of the lower arch of the device (Figure 2). It was important to determine if the increased size adversely affected comfort, compliance, lip seal, and other clinical factors that are critical to treatment success. Though a few patients did comment on the slight increase in bulk from the point of view of comfort, the addition of the chip did not adversely affect the outcome of the study or the therapy.

Compliance data were acquired for each patient during follow-up appointments by placing the device on the compliance recorder base station, which transferred the data from the chip to an online report provided by the chip manufacturer. The data transfer process worked as expected. No issues were observed by the clinical team. Reading the data via the online report was straightforward. The report tracked nightly compliance data, as well as aggregate performance data throughout the treatment period. 
Figure 2-Oral appliance with compliance recording chip.

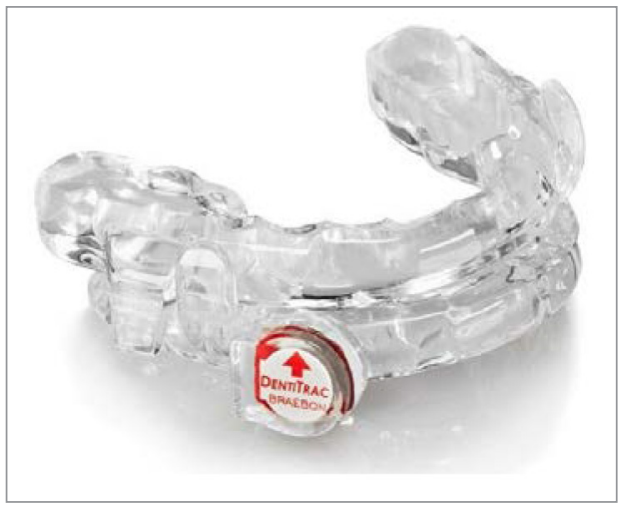

Pictured is the ProSomnus IA Sleep Device (ProSomnus Sleep Technologies, Pleasanton, California, United States) with a compliance recorder (Dentitrac, Braebon Medical Corporation, Kanata, Ontario, Canada).

It is worth noting that this OAT-CR uses a novel method for embedding the compliance recorder. Other OAT-CR use a traditional, cold cure additive method to affix the compliance recorder to the splint aspect of the OAT device. It is thought among practitioners that the cold cure method may adversely affect recorder performance by unintentionally subjecting the chip to thermal and physical stresses. The OAT-CR utilized in this study uses a forward engineering method to prospectively design a pocket for the chip into the design of the oral appliance. This approach mitigates the risk of exposing the chip to these potentially detrimental stresses, and ensures that the chip is affixed to the device according to the manufacturer's dimensional specifications. Each of the compliance recorders survived the duration of the study, and functioned as intended.

The objectively recorded data from this pilot study affirmed prior reports of OAT compliance rates. The $87.9 \%$ compliance rate in the current study was $87.9 \%$. Barnes and colleagues ${ }^{16}$ found that $43 \%$ of patients on CPAP and $76 \%$ of patients on OAT met a minimum compliance definition. Vanderveken et al. ${ }^{10}$ reported an oral appliance compliance rate of $84 \%$ using objectively recorded data and a similar definition for compliance (Figure 3).

Mean hours in therapy per night is a commonly utilized metric for quantifying treatment compliance. Four hours per night is considered a minimum standard for treatment compliance; however, other studies have suggested that therapeutic benefits increase commensurate with longer nightly usage. ${ }^{7}$

The mean use, objectively recorded in the current study, was $7.4 \pm 1.4 \mathrm{~h} /$ night. This finding is slightly higher than the $6.6 \pm 1.3 \mathrm{~h} /$ night reported in a study by Vanderveken et al. 10 that utilized objectively recorded compliance data. These data are likely statistically similar to the current study given the sample sizes, mean values, and standard deviations of the studies. The randomized, controlled, crossover study by Barnes et al. ${ }^{16}$ reported average usage of $3.6 \pm 0.3 \mathrm{~h} /$ night for CPAP and $5.5 \pm 0.3 \mathrm{~h} / \mathrm{night}$ for OAT. However, it is important to note that the study utilized objectively recorded compliance

\section{Figure 3-Compliance rate comparison.}

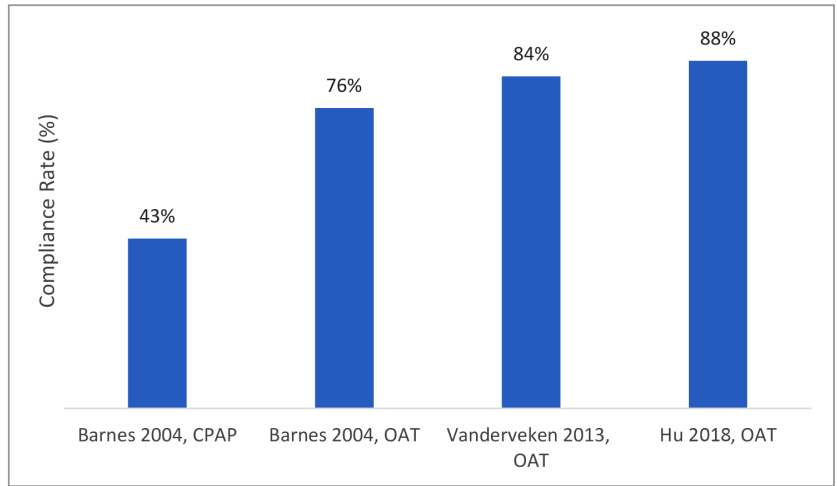

CPAP = continuous positive airway pressure, OAT = oral appliance therapy.

Figure 4-Nightly use comparison.

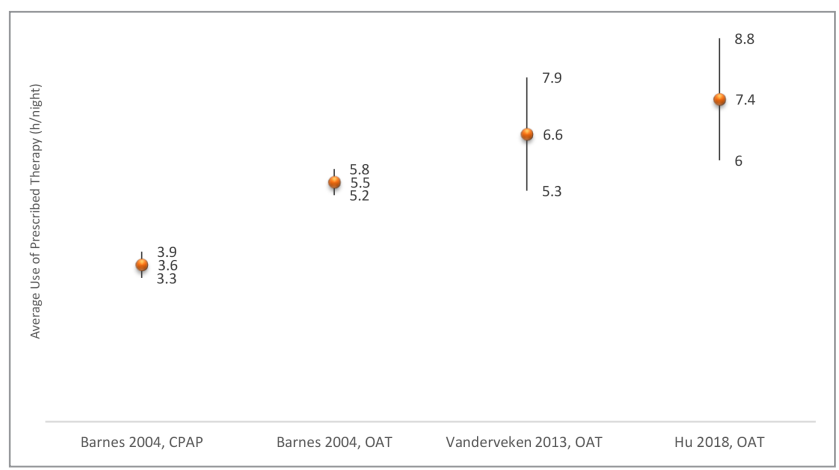

Values shown are mean hours \pm 1 standard deviation.

CPAP = continuous positive airway pressure, OAT = oral appliance therapy.

data for CPAP, but relied on patient journal data for oral appliances (Figure 4).

A latent intent of this feasibility study is to broaden the foundation for further research on the topic of OAT compliance rates. By reporting data on the feasibility and performance of this OAT-CR, the authors hope to encourage future research that addresses the limitations of this study, including: larger sample sizes, longer time durations, controlled compliance studies comparing OAT devices and CPAP devices, and more reporting, in general, of data from OAT-CR.

In conclusion, this pilot study establishes the clinical feasibility of a new OAT-CR for the treatment of OSA. The results indicate that the new OAT-CR performs as intended, and was accepted by study participants. The objectively recorded compliance data from this investigation affirm findings from prior studies with respect to the compliance rates and mean hours of nightly use associated with OAT.

\section{ABBREVIATIONS}

AHI, apnea-hypopnea index

BMI, body mass index 
CPAP, continuous positive airway pressure

FDA, United States Food and Drug Administration

OAT, oral appliance therapy

OAT-CR, oral appliance therapy device with compliance recorder

OSA, obstructive sleep apnea

\section{REFERENCES}

1. Young T, Finn L, Peppard PE, et al. Sleep disordered breathing and mortality: eighteen-year follow-up of the wisconsin sleep cohort. Sleep. 2008;31(8):1071-1078.

2. Ravesloot MJ, de Vries N, Stuck BA. Treatment adherence should be taken into account when reporting treatment outcomes in obstructive sleep apnea. Laryngoscope. 2014;124(1):344-345.

3. Weaver TE, Maislin G, Dinges DF, et al. Relationship between hours of CPAP use and achieving normal levels of sleepiness and daily functioning. Sleep. 2007;30(6):711-719.

4. Kribbs NB, Pack AI, Kline LR, et al. Effects of one night without nasal CPAP treatment on sleep and sleepiness in patients with obstructive sleep apnea. Am Rev Respir Dis. 1993;147(5):1162-1168.

5. McEvoy RD, Antic NA, Heeley E, et al. CPAP for prevention of cardiovascular events in obstructive sleep apnea. $N$ Engl J Med. 2016;375(10):919-931.

6. Kribbs NB, Pack AI, Kline LR, et al. Objective measurement of patterns of nasal CPAP use by patients with obstructive sleep apnea. Am Rev Respir Dis. 1993;147(4):887-895.

7. Sutherland K, Phillips CL, Cistulli PA. Efficacy versus effectiveness in the treatment of obstructive sleep apnea: CPAP and oral appliances. Journal of Dental Sleep Medicine. 2015;2(4):175-181.

8. White DP, Shafazand S. Mandibular advancement device vs CPAP in the treatment of obstructive sleep apnea: are they equally effective in short term health outcomes? J Clin Sleep Med. 2013;9(9):971-972.

9. Ramar K, Dort LC, Katz SG, et al. Clinical practice guideline for the treatment of obstructive sleep apnea and snoring with oral appliance therapy: an update for 2015. Journal of Dental Sleep Medicine. 2015;2(3):71-125.

10. Vanderveken OM, Dieltjens M, Wouters K, De Backer WA, Van de Heyning PH, Braem MJ. Objective measurement of compliance during oral appliance therapy for sleep-disordered breathing. Thorax. 2013;68(1):91-96.
11. Inoko Y, Yoshimura K, Kato C, et al. Efficacy and safety of temperature data loggers in measuring compliance with the use of oral appliances. Sleep Biol Rhythms. 2009;7:188-192.

12. Lowe AA, Sjoholm TT, Ryan CF, Fleetham JA, Ferguson KA, Remmers JE. Treatment, airway and compliance effects of a titratable oral appliance. Sleep. 2000;23(Suppl 4):S172-S178.

13. United States Food and Drug Administration website. 510(k) Premarket Database. Accessed December 11, 2017.

14. Fleury B, Lowe AA, ORal Appliance Network for Global Effectiveness Group. Current barriers and study needs for oral appliance therapy: the personal perspective of a physician and dentist. Journal of Dental Sleep Medicine. 2014;1(3):123-127.

15. Charkhandeh S, Kuhns D, Kim S. A fully digital workflow and device manufacturing for mandibular repositioning devices for the treatment of obstructive sleep apnea: a feasibility study. Journal of Dental Sleep Medicine. 2017;4(4):97-102.

16. Barnes M, McEvoy RD, Banks S, et al. Efficacy of positive airway pressure and oral appliance in mild to moderate obstructive sleep apnea. Am J Respir Crit Care Med. 2004;170(6):656-664.

\section{SUBMISSION \& CORRESPONDENCE INFORMATION}

Submitted for publication October 31, 2017

Submitted in final revised form December 11, 2017

Accepted for publication January 3, 2018

Address correspondence to: Len Liptak, MBA, 5860 West Las Positas Blvd, Suite 25, Pleasanton, CA 94588; Tel: (925) 353-7904; Email: 1liptak@ prosomnus.com

\section{DISCLOSURE STATEMENT}

Work for this study was performed at Dr. Jerry Hu Family Dentistry, LLC, Soldotna, Alaska. Dr. Jerry Hu and Mr. Len Liptak have seen and approved the manuscript. This study was supported by ProSomnus Sleep Technologies. Dr. Jerry Hu reports no other conflicts of interest. Len Liptak is CEO of ProSomnus Sleep Technologies. 